\title{
Specific Ion Effects on the Water Solubility of Macromolecules
}

\author{
Yanjie Zhang, Steven Furyk, David E. Bergbreiter, ${ }^{*}$ and Paul S. Cremer ${ }^{*}$ \\ Department of Chemistry, Texas A\&M University, College Station, TX 77843 \\ E-mail: cremer@mail.chem.tamu.edu; bergbreiter@mail.chem.tamu.edu
}

\section{Supporting Information}

\section{Preparation and fractionation of PNIPAM}

PNIPAM was obtained by free-radical polymerization of its monomer. A solution of $N$-isopropylacrylamide and 2,2'-azoisobutylnitrile (AIBN) in methanol was degassed and lowered into a preheated oil bath at $70{ }^{\circ} \mathrm{C}$ under positive pressure of $\mathrm{N}_{2}$. After stirring for $25 \mathrm{~h}$, the solvent was removed and the resulting solid was dried under vacuum. The polymer was purified from any remaining monomer by first dissolving it in tetrahydrofuran (THF) and then precipitating the polymer into hexanes three times. Because it was deemed desirable to use polymer material with a low polydispersity, the nascent PNIPAM was dried, redissolved in acetone $(10 \mathrm{~g} / \mathrm{L})$, and precipitated stepwise by addition of hexanes. ${ }^{1}$ This gave rise to four fractions with molecular weights of $4.75 \times$ $10^{5}, 1.70 \times 10^{5}, 5.58 \times 10^{4}$, and $1.78 \times 10^{4} \mathrm{~g} / \mathrm{mol}$ as determined by static light scattering in methanol. The $1.70 \times 10^{5} \mathrm{~g} / \mathrm{mol}$ sample was employed in all experiments described herein. 
The fractionated samples described above were analyzed by gel permeation chromatography in THF. This analysis used $3.48 \mathrm{mg}$ of the PNIPAM fraction with $M_{\mathrm{w}}=$ $1.7 \times 10^{5}$ Da dissolved in degassed THF and was carried out using a Viscotek IMBMMW-3078 mixed bed column using a Viscotek GPC. The instrument was equipped with multiple detectors including a Model VE 3580 RI detector and OmniSEC software. The results indicated that the polydispersity of the fractionated PNIPAM sample used in the LCST studies described above was 1.32 based on polystyrene standards as a reference.

\section{Sample preparation}

All chemicals used in these experiments including inorganic salts and $\mathrm{D}_{2} \mathrm{O}$ were purchased from Aldrich. Low-conductivity $\mathrm{H}_{2} \mathrm{O}$, produced from a NANOpure Ultrapure Water System (Barnstead, Dubuque, IA) with a minimum resistivity of $18 \mathrm{M} \Omega \cdot \mathrm{cm}$, was used to prepare the polymer and salt solutions. Stock solutions of PNIPAM at $20 \mathrm{mg} / \mathrm{mL}$ were prepared by allowing the polymer to dissolve overnight in $\mathrm{H}_{2} \mathrm{O}$ or $\mathrm{D}_{2} \mathrm{O}$ at $4{ }^{\circ} \mathrm{C}$. Aliquots of these solutions were added to water or inorganic salt solutions at the desired concentration. The final polymer concentration for all experiments performed herein was $10 \mathrm{mg} / \mathrm{mL}$.

\section{Fabrication of a temperature gradient device and measurement of LCSTs}

Fabrication of our temperature gradient device has been described previously. ${ }^{2-4}$ Briefly, a cover glass acting as both the sample stage and heat conductor was mounted on top of two parallel brass tubes (1/8 in. wide, K\&S Engineering, Chicago, IL) through 
which hot and cold antifreeze solutions could be individually flowed using standard water bath circulators (Fisher Scientific, Pittsburgh, PA). The distance between the brass tubes was approximately $5 \mathrm{~mm}$. Rectangular borosilicate capillary tubes (VitroCom, Inc.) with dimensions of $2 \mathrm{~cm} \times 1 \mathrm{~mm} \times 100 \mu \mathrm{m}$ (length $\times$ width $\times$ height) were used as sample containers and placed parallel to the temperature gradient. Light scattering from polymer solutions was monitored via a CCD camera (Micromax 1024, Princeton Instruments) using dark field optics under an inverted microscope (Nikon, TE2000-U). Two different polymer solutions with previously measured LCSTs served to calibrate the slope of the gradient in each experiment. ${ }^{2-4}$ The first control solution was $10 \mathrm{mg} / \mathrm{mL}$ PNIPAM in $\mathrm{H}_{2} \mathrm{O}$ with a known LCST of $30.19 \pm 0.05^{\circ} \mathrm{C}$ and the second was $10 \mathrm{mg} / \mathrm{mL}$ PNIPAM in $0.7 \mathrm{M} \mathrm{KCl}$ with a known LCST of $25.96 \pm 0.05^{\circ} \mathrm{C}$. Six rectangular capillary tubes could be fit side-by-side under a $\times 2$ objective. The temperature along the capillary tubes was determined by counting pixels in a linescan drawn along the direction of the gradient in the corresponding CCD images. The scattering intensity at each temperature was determined by averaging data from a $635 \mu \mathrm{m}$ long slice ( 50 pixels) perpendicular to the linear temperature gradient (Figure S1). There were $253 \pm 2$ pixels parallel to the temperature gradient between 22.0 and $32.0^{\circ} \mathrm{C}$. The exact number of pixels over the 10 ${ }^{\circ} \mathrm{C}$ range was determined independently for each sample with the aid of the two known calibration standards. Each slice represented slightly less than $0.040{ }^{\circ} \mathrm{C}$.

The light scattering intensities vs. temperatures curves shown in this paper were obtained by a three point running average of the raw data. LCST values were taken as the initial break points ${ }^{5}$ of these light scattering intensity versus temperature curves. This was simply the intersection of the initial slope of the scattering curve vs. the slope of the 
scattering curve in the middle of the transition. The measurement of the LCST at each salt concentration was repeated at least 8 times and the mean value was taken. The measurements had a typical standard error of $\pm 0.05^{\circ} \mathrm{C}$.

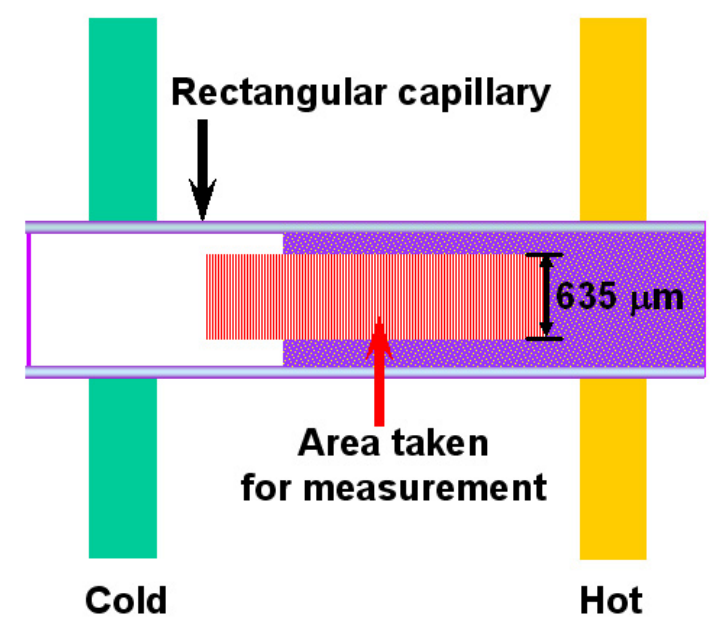

Figure S1. Schematic diagram of the linear temperature gradient used to obtain light scattering curves. The PNIPAM solution inside the capillary tube shows no light scattering at low temperature (white region inside the tube), but scatters light strongly above the lower critical solution temperature (purple region inside the tube). Data were taken in slices (red vertical lines) from the middle of each tube and the scattering intensity was averaged over a $635 \mu \mathrm{m}$ line at each temperature.

Experiments were performed to insure all data were taken under equilibrium conditions. For example, the capillary tubes were removed from the gradient, flipped over, and reinstalled. Also, individual capillary tubes were pushed back and forth parallel to the temperature gradient. These experiments showed that the polymer folding process was completely reversible and the onset point of the LCST was always found to be at the 
same location. In fact, equilibrium must be established under the conditions of these experiments in no more than a few seconds, which simply represented the minimum time needed to move a tube and retake the data.

All measurements were made in solutions containing only the salts indicated. No buffers were added as this would also change the salt composition of the solution. This choice had a couple of consequences that merit discussion. First, $\mathrm{CO}_{2}$ in the air will slowly become dissolved in the solution. At equilibrium approximately $12 \mu \mathrm{M} \mathrm{CO}_{2}$ will be dissolved assuming the atmosphere contains $33 \mathrm{~Pa}$ of $\mathrm{CO}_{2}$ with a Henry's constant of $1.50 \times 10^{8} \mathrm{~Pa}$ for $\mathrm{CO}_{2}$ over water at $20{ }^{\circ} \mathrm{C} .{ }^{6}$ The measured $\mathrm{pH}$ of our purified water was 5.8. Second, some of the individual salts will affect the $\mathrm{pH}$ of the solution. The greatest $\mathrm{pH}$ changes for the salts used here involved $\mathrm{Na}_{2} \mathrm{CO}_{3}$ and $\mathrm{NaH}_{2} \mathrm{PO}_{4}$. At the highest concentration of $\mathrm{Na}_{2} \mathrm{CO}_{3}$ employed, the $\mathrm{pH}$ of the solution was 12.1. On the other hand, the $\mathrm{pH}$ of the $0.6 \mathrm{M} \mathrm{NaH}_{2} \mathrm{PO}_{4}$ solution was 4.3. Control experiments were performed at different $\mathrm{pH}$ values to determine the effect of $\mathrm{pH}$ on the LCST of PNIPAM. This was done by titrating $\mathrm{H}_{3} \mathrm{PO}_{4}$ with $\mathrm{NaOH}$ under conditions where the concentration of phosphate ions never exceeded $10 \mathrm{mM}$. The results showed that the LCST was unchanged within experimental error between $\mathrm{pH} 4$ and 12. Finally, it was necessary to consider the identity of the anion in solution for those with titratable groups and keep it identical over the entire concentration range probed. For example, the $\mathrm{pK}_{\mathrm{a}}$ of $\mathrm{HCO}_{3}{ }^{-}$is 10.3. Even at the lowest concentration of $\mathrm{Na}_{2} \mathrm{CO}_{3}$ employed (.033 M), the $\mathrm{pH}$ was 11.8 and therefore the fraction of $\mathrm{CO}_{3}{ }^{2-}$ was $97 \%$. Also, the dominant form of phosphate in these experiments was $\mathrm{HPO}_{4}{ }^{2-}$ and always exceeded 99.8\%. 


\section{References}

(1) Fujishige, S. Polymer J. 1987, 19, 297-300.

(2) Mao, H. B.; Li, C. M.; Zhang, Y. J.; Bergbreiter, D. E.; Cremer, P. S. J.

Am. Chem. Soc. 2003, 125, 2850-2851.

(3) Zhang, Y. J.; Mao, H. B.; Cremer, P. S. J. Am. Chem. Soc. 2003, 125,

15630-15635.

(4) Mao, H. B.; Li, C. M.; Zhang, Y. J.; Furyk, S.; Cremer, P. S.; Bergbreiter, D. E. Macromolecules 2004, 37, 1031-1036.

(5) Schild, H. G.; Tirrell, D. A. J. Phys. Chem. 1990, 94, 4352-4356.

(6) Carroll, J. J.; Mather, A. E. J. Solution Chem. 1992, 21, 607-621. 